\title{
Estimation of CNC Grinding Process Parameters Using Different Neural Networks
}

\author{
Tomislav ŠARIĆ, Goran ŠIMUNOVIĆ, Đorđe VUKELIĆ, Katica ŠIMUNOVIĆ, Roberto LUJIĆ
}

\begin{abstract}
Continuation of research on solving the problem of estimation of CNC grinding process parameters of multi-layer ceramics is presented in the paper. Heuristic analysis of the process was used to define the attributes of influence on the grinding process and the research model was set. For the problem of prediction - estimation of the grinding process parameters the following networks were used in experimental work: Modular Neural Network (MNN), Radial Basis Function Neural Network (RBFNN), General Regression Neural Network (GRNN) and Self-Organizing Map Neural Network (SOMNN). The experimental work, based on real data from the technological process was performed for the purpose of training and testing various architectures and algorithms of neural networks. In the architectures design process different rules of learning and transfer functions and other attributes were used. RMS error was used as a criterion for value evaluation and comparison of the realised neural networks and was compared with previous results obtained by Back-Propagation Neural Network (BPNN). In the validation phase the best results were obtained by Back-Propagation Neural Network (RMSE 12,43 \%), Radial Basis Function Neural Network (RMSE 13,24 \%,), Self-Organizing Map Neural Network (RMSE 13,38 \%) and Modular Neural Network (RMSE 14,45\%). General Regression Neural Network (RMSE 21,78 \%) gave the worst results.
\end{abstract}

Keywords: algorithms of neural networks; CNC grinding; estimation; prediction

\section{INTRODUCTION}

Implementation and realisation of Industry 4.0 requires designing also, among other things, a part of the "smart" support to the design of technological processes. In this segment, the approach known as "soft computing" can be of a considerable use. It includes various approaches and the application of artificial intelligence methods. As the influence of market to the terms of running a business is considerable, firms must be able to react by their organization, intellectual capacity and up-to-date processing systems. Departments for the preparation and design of technological processes have an important place and role in any manufacturing process. The designed technological processes with the corresponding documentation must make sure the strict and rigorous conditions of the market focused on price, quality, terms and time of delivery and other attributes are being met. The technological processes, among other things, also include different grinding processes. To help design the technological processes of grinding various approaches can be used, some of them being "soft computing", statistic, mathematic etc. A number of authors use the methodology of "soft computing" in their researches of the grinding process. Some of them publish them in scientific papers [1-3]. An important part of the published papers in which the methodology of "soft computing" is used as a dominant technique, apply artificial neural networks and/or other approaches (fuzzy logic, evolutionary computation) - based on the origin of species, such as: Genetic Algorithm, Swarm Intelligence, Ant Colony Optimizations, machine learning and others. In grinding processes, authors intensively use neural networks, the problems of prediction and classification being modelled most often. By investigating grinding problems in different fields, the problems raised through investigating prediction models prevail in various papers. Thus, authors [4] use the response surface methodology model along with artificial neural networks. The applied approaches with acquired results expressed in predictive values are compared with experimental results in designed models showing there is no considerable departure. In paper [5] authors describe the nonlinearity definition problem with regard to the relation between the precision of the grinding process and the processing parameters by using conventional experimental methods. Combination of different approaches is suggested as a solution. Diverse parameters affecting the precision of the grinding process on a particular machine are analysed first, then an experiment for technological data collection is designed. The approach based on "support vector machine-SVM" is suggested for the process modelling in combination with particle swarm optimization - PSO as well as the neural network approach (Back Propagation BP algorithm).

The results showed that the estimation model based on the PSO - SVM combination is quicker and more precise than the neural network algorithm with the BP algorithm for the set model. Back Propagation Neural Network BPNN algorithm - is also used as a basis for the research in paper [6] in the rotary ultrasonic grinding machining process. However, it is found that the nonlinear relationship of the model is very complex and the traditional BPNN algorithm cannot give satisfactory results. An improved algorithm of BPNN is suggested based on the Powel method.

Theoretical bases with a review of the calculation are given in the paper. Acquired results confirmed validity of development and usage of the algorithm model improved through higher speed and better accuracy of iterations. BPNN is also used in paper [7] for the problem of prediction in combination with the multiple linear regression (MLR) method. A study model is set up with characteristic attributes and in both approaches the set of data for learning and testing gave the result of $2,79 \%$ in the learning phase and $4,18 \%$ in the validation phase for BPNN. Analysis of obtained results demonstrated that the BPNN and MLR approaches can be used with acceptable exactness. In paper [8] authors apply artificial neural networks for evaluation of the grinding process.

The other group of papers on grinding processes includes investigations that use neural networks in solving classification problems. In machining processes, exotic materials demanding high quality treatment and narrow tolerances are being increasingly used [9]. In order to meet 
the demanded requirements there is an increasing need for understanding the grinding process models. For this reason the unit events of material interaction in grinding are investigated. The process involves: rubbing, ploughing and cutting. Neural networks are used for the classification and verification checking of Grit (SG) phenomena. After the cutting, ploughing and rubbing they gave high confidence to the exactness of classification results. Interesting output results were obtained that correlated for both classifiers, which means there is higher usability of cutting than of ploughing and rubbing because interaction between sand and workpiece is becoming more and more important (measured depth of cut increases).

The problem of tool wear prediction is an important parameter for a rational planning and control of manufacturing processes. Paper [10] models and investigates this problem using different neural network algorithms (Back Propagation and Self-organizing map) and k-means clustering algorithm for noise filtration. Using different algorithms of neural networks i.e. their upgrading or combination with other approaches is investigated in the process of grinding with high temperature problems during the treatment [11]. The paper depicts the research on prediction of high-speed grinding temperature using different approaches: BPNN in combination with particle swarm optimization (PSO) algorithm, BPNN in combination with gradient descent method (GD), BPNN in combination with LevenbergMarquardt (LM) algorithm. The results of the research show that the first approach (BPNN with PSO) has an advantage with regard to speed of convergence, precision of adaptation and exactness of prediction over the other two approaches (BPNN with GD method and BPNN with LM algorithm) in prediction of grinding temperature. In papers [12-15] authors investigate and model production problems and maintenance planning problems and use different algorithms of neural networks aimed at predicting model variables. The following algorithms of neural networks were used in investigations: Back-Propagation Neural Network (BPNN), Modular Neural Network (MNN) and RadialBasis Function Neural Network (RBFNN), General Regression Neural Network (GRNN) and Self-Organizing Map Neural Network (SOMNN).

Therefore in all the mentioned papers conclusions in most cases justify the application of neural networks (ANN) in investigating different production-technological problems. This paper continues the investigations given in paper [2] so that on the set model and the experimental results diverse algorithms of neural networks are used to model and predict output parameters of the technological process CNC grinding of multi-layer ceramics.

\section{METHODOLOGY AND GOAL OF INVESTIGATION}

A short review of the technological process $\mathrm{CNC}$ grinding of workpieces modelled from multi-layer ceramics is given in paper [2]. The workpieces are formed from cca. 60 layers of sintered ceramics of $7 \times 7$ millimetres cross-section and of length 33 millimetres (Fig. 1). In exploitation the function of these elements is to extend by cca. 0,05 millimetres under the action of electric current. In production there are special requirements on dimensional tolerances and the tolerances of shape (parallelism). As the actual study presents continuation of investigations, a short description and proposition of the investigation will be submitted as follows. Grinding of multilayer ceramics is performed on CNC grinding machines type WAC175 SCALAR (producer Wendt). Within its workspace this grinding machine integrates a SCARA robot with four degrees of freedom of motion (Fig. 2). Main function of the robot is manipulation of workpieces.
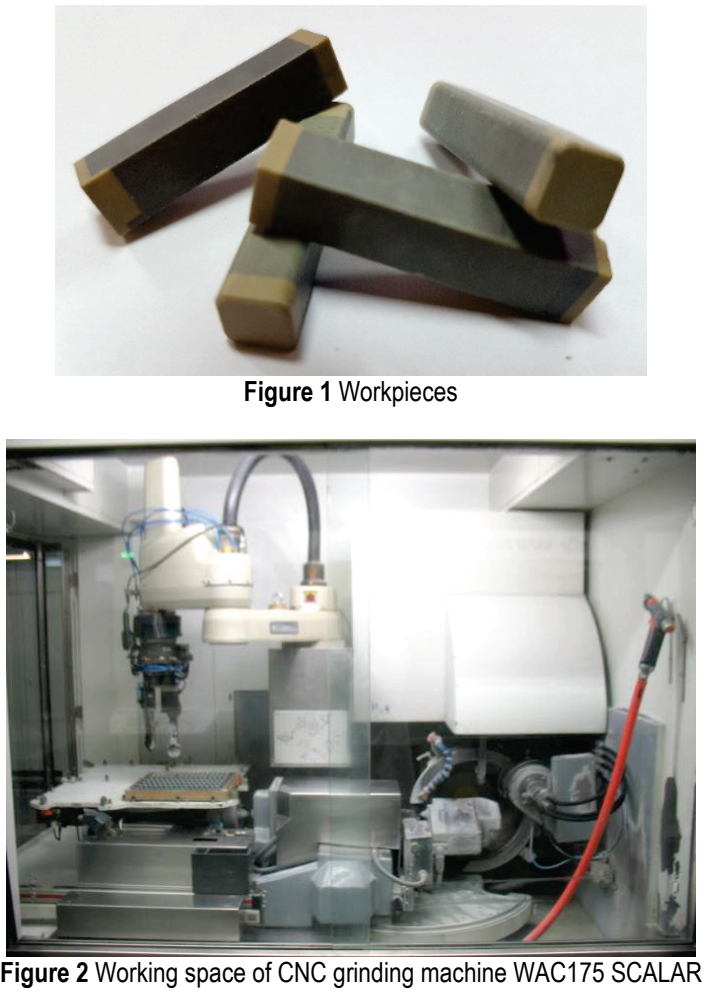

The problem and aim of the investigation was to control the dependencies of the variables that affect plane parallelism (PPL) of workpieces and their changes. The study is based on the data collected from data base of the technological process of grinding. In experimental part of the study different algorithms of neural networks and different algorithms of learning (and other attributes of neural networks) will be used so as to model the processes of grinding depending on the observed variables (machine, operator, foil and "hori" - lines) affecting the plane parallelism of workpieces.

\subsection{Hypotheses and Experimental Work Modelling of the Process}

Based on the established model of grinding process and on technological experience acquired in the machining process as well as on heuristic approach the study model is defined. Input variables are defined for the set model vector $\boldsymbol{X}_{\mathrm{i}}=\{$ machine, operator, foil and hori-line $\}$ as well as output variable - vector $\boldsymbol{Y}_{\mathrm{o}}=\{$ plane parallelism $-P P L\}$.

The selected variables are shortly described, as follows:

- Machine - different machines in production lines can have different deviations of influence on PPL. According to the control protocol, the machine measures the length of the workpiece before and after the operation of grinding. If the workpiece is outside 
the permitted tolerance limits, the grinding process stops. During grinding, deviations in the shape of workpieces can occur, although within the geometry tolerances (length). In this case, the grinding process results in workpieces in the form of parallelograms, which is geometrically inadequate (a reject).

- The operator of machine has an important role for the accuracy of PPL. He performs the PPL control measurements and based on obtained measures he must decide on eventual corrective actions on machine (adjustments). The operator confirms the performed activities of control (measurement) on workpieces by his own identification.

- Foil (type of ceramic foil - batch) represents the layers of a workpiece. If the electrodes are not printed adequately on the ceramic foil during production or if the foil is not well positioned, it can lead to damaged workpieces (rejects).

- Hori-line represents the production line on which a ceramic block is formed. Several errors may occur on this line, such as: inadequately folded foil, inadequate temperature of sintering and sintering pressure, inadequate temperature of firing and incorrectly cut parts. If the technological parameters are not set correctly during grinding, the workpieces might be bent and adjusted by the grippers during the process of grinding, but after grinding and releasing of the grippers, the workpiece might return to the initial state and have inadequate PPL.

- Plane parallelism - PPL is defined as output variable of the model. The parallelism is measured in four points. If the difference between the maximum and minimum measure is within the tolerance limits, then the workpiece is acceptable and correct, otherwise it is not acceptable and represents a reject. Figure 3 shows the workpiece and the attributes of measuring in characteristic points.

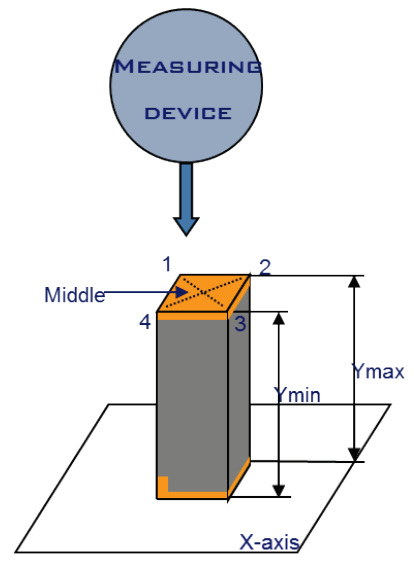

Figure 3 Characteristic points for PPL measuring [2]

For the selected variables of the studied model, the data are gathered from the technological process. Each of the variables is defined with the maximal and minimal values (Tab. 1).

The set of data for experimental work with different algorithms of neural networks is based on real data with a sample of 1860 cases. The data set is divided into three groups according to the output variable, following the principle: $60 \%$ for learning, $20 \%$ for testing and $20 \%$ for validation.

Table 1 Min-Max values of the model variables

\begin{tabular}{|c|c|c|c|}
\hline & \multirow{2}{*}{ MODEL VARIABLES } & \multicolumn{2}{|c|}{ VARIABLE VALUE } \\
\hline & & Min & Max \\
\hline \multirow{4}{*}{$\boldsymbol{X}_{\mathrm{i}}$} & Machine & 1 & 2 \\
\hline & The operator of machine & 1 & 11 \\
\hline & Foil & 3 & 10 \\
\hline & Hori-line & 1 & 3 \\
\hline $\boldsymbol{Y}_{\mathrm{o}}$ & Plane parallelism - PPL & 0 & 9 \\
\hline
\end{tabular}

The designed samples of data are used in experimental work for each designed architecture of neural network. Designing of the neural networks' diverse architectures is visible in the search for optimal attributes. For each designed architecture of a neural network with a defined algorithm the process of learning is conducted with testing and validation. The process of research and the experimental work are reflected in an iterative procedure and realization of dependency of particular attributes on the required output of the set model. As a criterion for acceptance of successful design of the neural networks' architectures the criterion of $R M S$ error is selected (Root Mean Square error - RMSE), given by the expression (1):

$R M S=\sqrt{M S}=\sqrt{\frac{\sum_{n=1}^{N}\left(d_{n}-y_{n}\right)^{2}}{N}}$

where: $M S$ - Mean Square error; $N$ - Number of pairs of the training set input-output values; $y_{n}$ - Neural network $n^{\text {th }}$ output; $d_{n}$ - Desired value of the neural network; $n^{\text {th }}$ output.

\subsection{A Short Review of the Selected Neural Networks with Obtained Results}

In this section, results are presented of five selected algorithms of neural networks: Back-Propagation Neural Network (BPNN), Modular Neural Network (MNN), Radial Basis Function Neural Network (RBFNN), General Regression Neural Network (GRNN) and Self-Organizing Map Neural Network (SOMNN). The results are obtained during experimental work on designing each particular architecture, i.e. adjustment of attributes (learning rules, transfer functions, coefficient of learning, momentum, size of epoch etc.) of neural networks. Only the most successful architectures are demonstrated by tables with RMS error produced.

Description of each particular network with its characteristics is not given in the paper due to its size. More about different architectures and their description can be found in various references.

\subsubsection{Back-Propagation Neural Network}

Back-Propagation Neural Network (BPNN) is a popular and often used network in scientific investigations and in the paper [2] that preceded the here presented investigation it also served as a basis. Neural network with one hidden layer and given attributes was used in this paper [2]. After designing a greater number of the neural networks' architectures, varying different attributes and 
rules of learning, the neural network with the least mean square error $0,1076(10,76 \%)$ in the learning (training) phase was adopted. Table 2 gives a review of the results in the learning phase with various transfer functions and rules (algorithms) of learning.

Table 2 BPNN - RMS error (Learning rule - Transfer function)

\begin{tabular}{|l|c|c|c|c|}
\hline \multicolumn{5}{|c|}{ Back-Propagation Neural Network (BPNN) } \\
\hline \multirow{2}{*}{ Transfer function } & \multicolumn{4}{|c|}{ Learning rule } \\
\cline { 2 - 5 } & DBD & Delta & Ext. DBD & Norm.D \\
\hline Linear & 0,3006 & 0,2906 & 0 & 0,2976 \\
\hline Sigmoid & $\mathbf{0 , 1 0 7 6}$ & 0,1111 & 0,1128 & 0,1101 \\
\hline Sinus & 0,3008 & 0,2907 & 0,7524 & 0,2983 \\
\hline Hyper.-Tan. & 0,3004 & 0,2906 & 0,3911 & 0,2973 \\
\hline
\end{tabular}

\subsubsection{Modular Neural Network}

Modular Neural Network(MNN) is composed of groups of neural networks connected into a whole while individual neural networks are by synergism focused onto the segments of the established problem and represent "local experts". These "local experts" are connected by a transitory network that conducts the segmentation of the studied problem and the learning. If it should turn out that only one neural network is good for solving the designed problem then the transitory network will give preference to only one neural network among the established "local experts". The modular neural network's learning takes place simultaneously for transitory network and for "local experts". By modelling and experimental work with this network, after adjusting the network attributes (by varying transfer functions and rules of learning and other attributes), results are obtained with the least mean square root of $0,1352(13,52 \%)$ in the neural network's phase of learning (training). Tab. 3 displays the results achieved in the phase of learning with diverse transfer functions and rules (algorithms) of learning.

Table 3 MNN - RMS error (Learning rule - Transfer function)

\begin{tabular}{|l|c|c|c|c|}
\hline \multicolumn{5}{|c|}{ Back-Propagation Neural Network (BPNN) } \\
\hline \multirow{2}{*}{ Transfer function } & \multicolumn{4}{|c|}{ Learning rule } \\
\cline { 2 - 5 } & DBD & Delta & Ext. DBD & Norm.D \\
\hline Linear & 0,3195 & 0,3817 & 0 & 0,3717 \\
\hline Sigmoid & $\mathbf{0 , 1 3 5 2}$ & 0,1387 & 0,1401 & 0,1378 \\
\hline Sinus & 0,3680 & 0,3814 & 0,3641 & 0,3698 \\
\hline Hyper.-Tan. & 0,3688 & 0,3822 & 0,3819 & 0,3620 \\
\hline
\end{tabular}

\subsubsection{Radial Basis Function Neural Network}

Radial Basis Function Networks (RBFNN) can in most general cases include any network that inside the neuron in the hidden layer has a radially symmetric transfer function. Learning in a network with radial basis functions can be defined by layers. Learning starts in input layer on the principle "without a teacher". As a result the position of the centre is defined. In output layer the calculation finishes and in relation to the real outputs the error is defined. In the output and hidden layer learning is conducted on the principle "with a teacher". In experimental work with RBFNN, after adjusting the network attributes, the results with the least square root of $0,1132(11,32 \%)$ are obtained in the phase of the neural network learning (training). Tab. 4 gives a survey of results of RBFNN in the phase of learning with diverse transfer functions and rules (algorithms) of learning.
Table 4 RBFNN - RMS error (Learning rule - Transfer function) Back-Propagation Neural Network (BPNN)

\begin{tabular}{|l|c|c|c|c|}
\hline \multirow{2}{*}{ Transfer function } & \multicolumn{4}{|c|}{ Learning rule } \\
\cline { 2 - 5 } & DBD & Delta & Ext. DBD & Norm.D \\
\hline Linear & 0,3161 & 0,3076 & 0,3308 & 0,2940 \\
\hline Sigmoid & 0,1281 & $\mathbf{0 , 1 1 3 2}$ & 0,1189 & 0,1169 \\
\hline Sinus & 0,3160 & 0,3087 & 0,3281 & 0,2938 \\
\hline Hyper.-Tan. & 0,3163 & 0,3094 & 0,3321 & 0,2937 \\
\hline
\end{tabular}

\subsubsection{Self-Organizing Map Neural Network}

Self-Organizing Map Neural Network (SOMNN) is one of important architectures of neural networks. It is developed in the course of years between the Adalin network and the Hopfield network. The key difference between the SOMNN and other networks is in the fact that it learns without supervision and that is why the key word in the name of the network is self-organizing. SOMNN is usually used in combination with other layers of neural networks, notably for the problems of estimation and classification. The network begins to learn without supervision, then the supervision of learning (training) is required for the output layer. It is necessary for the network to have a necessary number of iterations so as to be able to stabilize. The experimental work with SOMNN gave the results with the least average square error of $0,1317(13,17$ $\%$ ) in the phase of the neural network learning (training). Tab. 5 gives a review of the SOMN results obtained in the phase of learning with diverse functions and rules (algorithms) of learning.

Table 5 SOMNN - RMS error (Learning rule - Transfer function)

\begin{tabular}{|l|c|c|c|c|}
\hline \multicolumn{5}{|c|}{ Back-Propagation Neural Network (BPNN) } \\
\hline \multirow{2}{*}{ Transfer function } & \multicolumn{4}{|c|}{ Learning rule } \\
\cline { 2 - 5 } & DBD & Delta & Ext. DBD & Norm.D \\
\hline Linear & 0,3477 & 0,3871 & 0,3925 & 0,3632 \\
\hline Sigmoid & 0,1655 & 0,1348 & 0,1364 & $\mathbf{0 , 1 3 1 7}$ \\
\hline Sinus & 0,3476 & 03791 & 0,4073 & 0,3628 \\
\hline Hyper.-Tan. & 0,3468 & 0,3733 & 0,3879 & 0,3624 \\
\hline
\end{tabular}

\subsubsection{General Regression Neural Network}

As a rule, it can be said that the General Regression Neural Network is used for solving the problems of prediction as well. Main advantages of GRNN are: quick learning, convergence to optimal regression if number of samples is large enough. It can be effective with even a smaller number of data. The experimental work with GRNN, after adjusting the network attributes, gave the results with the least square error of $0,2085(20,85 \%)$ in the phase of learning (training) of the neural network.

\subsection{Review of the Best Results with the Selected Neural Networks}

After carrying out the experimental work and designing numerous architectures for the purpose of modelling prediction with the selected neural networks in the phase of learning, tabular survey of results is given for each neural network (Tab. 2 - Tab. 5). The tables display the diverse used algorithms - rules of learning (Delta, Delta-Bar-Delta, Ext. Delta-Bar-Delta, Norm.-Cum. Delta) and transfer functions (Linear, Sigmoid, Sinus, Hyperbolic-Tangent). Following the experimental phase of neural networks learning, the next phase, validation, was 
carried out. In this phase a new dataset sample for validation was used. In the validation phase the weights of connections between neurons were not changed and the weights were adopted that were realised with the minimal RMSE. The validation phase results are shown in Tab. 6 along with the best results in the learning phase.

Table 6 Review of RMS error for diverse neural networks in the phase of learning and validation

\begin{tabular}{|c|l|c|c|}
\hline \multirow{2}{*}{ No. } & \multicolumn{1}{|c|}{ Type of Neural Network } & \multicolumn{2}{c|}{ Best Cases - NN phases } \\
\cline { 3 - 4 } & \multicolumn{1}{|c|}{$\begin{array}{l}\text { Learning } \\
\text { (Back-Propagation Neural Network }\end{array}$} & 0,1076 & 0,1243 \\
\hline 2 & Modular Neural Network (MNN) & 0,1352 & 0,1445 \\
\hline 3 & $\begin{array}{l}\text { Radial Basis Function Neural Network } \\
\text { (RBFNN) }\end{array}$ & 0,1132 & 0,1324 \\
\hline 4 & $\begin{array}{l}\text { General Regression Neural Network } \\
(\text { GRNN) }\end{array}$ & 0,2085 & 0,2178 \\
\hline 5 & $\begin{array}{l}\text { Self-Organizing Map Neural Network } \\
\text { (SOMNN) }\end{array}$ & 0,1317 & 0,1338 \\
\hline
\end{tabular}

Fig. 4 shows the best results obtained in investigation in designing diverse architectures of neural networks in the phases of learning and validation.

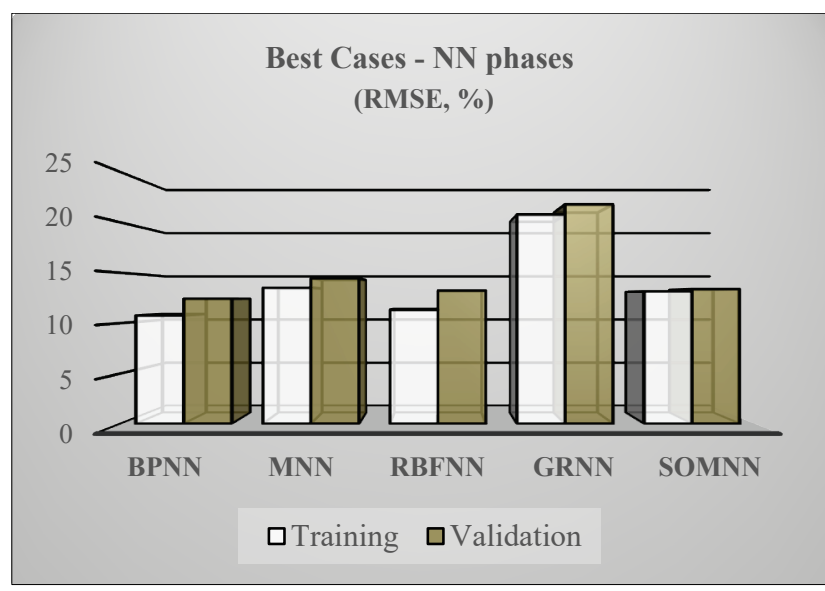

Figure 4 Results obtained by diverse neural networks

\section{CONCLUSION}

The paper is a continuation of the investigation into solving the parameter prediction problems in the grinding process of multilayer ceramics. The previously set investigation model and the results obtained by experimental work with Back-Propagation Neural Network are used in the study. Four additional neural networks are adopted: Modular Neural Network, Radial Basis Function Neural Network, General Regression Neural Network and Self-Organizing Map Neural Network. The continuation of investigation is conducted on the same dataset of technological data. In modelling the neural networks' diverse architectures, diverse rules (algorithms) of learning are used. Through experimental work the best architecture for every neural network is achieved by adjusting diverse attributes and adopting RMS error as a criterion for evaluation of the realised architectures. The set models of prediction based on five diverse neural networks gave the following results: BPNN RMS in the phase of learning $10,76 \%$, in the phase of validation $12,43 \%$; MNN in the phase of learning 13,52 $\%$, in the phase of validation $14,45 \%$; RBFNN in the phase of learning $11,32 \%$, in the phase of validation $13,24 \%$;
GRNN in the phase of learning $20,85 \%$, in the phase of validation $21,78 \%$; SOMNN in the phase of learning 13,17 $\%$, in the phase of validation $13,38 \%$. The obtained differences in error for all the five algorithms in the learning phase are up to $10,09 \%$ and up to $9,35 \%$ in the validation phase. The obtained difference in error with BPNN and RBFNN is at the level of $0,5 \%$, and with regard to $\mathrm{MNN}$ and SOMNN it is up to $2,7 \%$ with a notable difference of $10 \%$ in learning phase with regard to GRNN. The obtained levels of errors confirm the justifiability of a possible application of the set model. The obtained level of errors obtained with GRNN cannot provide justification for application.

The studies and achieved results in this field and continuation of investigations on other machining systems could serve as a basis for implementation of neural networks into design procedures of technological processes thus enabling easier work and providing help to technologists in production preparation departments.

\section{REFERENCES}

[1] Yadav, R.N. \& Yadava, V. (2015). Application of Soft Computing Techniques for Modeling and Optimization of Slotted-Electrical Discharge Diamond Face Grinding Process. Transactions of the Indian Institute of Metals, 68(5), 981-990. https://doi.org/10.1007/s12666-015-0536-6

[2] Šarić, T., Šimunović, G., Lujić, R., Šimunović, K., \& Antić, A. (2016). Use of Soft Computing Technique for Modelling and Prediction of CNC Grinding Process. Technical Gazette - Tehnički vjesnik, 23(4), 1123-1130. https://doi.org/10.17559/TV-20160405151333

[3] Li, Y., Cao, F., Wang, J. W., Zhang, G. F. Chen, X. Q., \& Chen, S. B. (2016). Modeling grinding process for difficultto-machine materials using artificial neural network. IEEE $11^{\text {th }}$ Conference on Industrial Electronics and Applications (ICIEA) / Hefei, P. R. China, 267-271.

[4] Unune, D. R. \& Mali, H. S. (2016). Artificial neural networkbased and response surface methodology-based predictive models for material removal rate and surface roughness during electro-discharge diamond grinding of Inconel 718 . Journal of Engineering Manufacture, 23(11), 2082-2091. https://doi.org/10.1177/0954405415619347

[5] Cai, H. X. \& Liu, W. (2017). A prediction method for the precision of extrusion grinding of a needle valve body. Production Engineering-Research and Development, 11(3), 295-305. https://doi.org/10.1007/s11740-017-0723-x

[6] Jing, J. T., Feng, P. F., Wei, S. L., \& Zhao, H. (2017). Investigation on surface morphology model of Si3N4 ceramics for rotary ultrasonic grinding machining based on the neural network. Applied Surface Science, 396, 85-94. https://doi.org/10.1016/j.apsusc.2016.11.044

[7] Azizi, A., Shafaei, S. Z., \& Rooki, R. (2016). Wear Rate Prediction of Grinding Media using BPNN and MLR Model in Grinding of Sulphide Ores. Iranian Journal of Materials Science and Engineering, 13(2), 73-84. https://doi.org/10.22068/ijmse.13.2.73

[8] Umucu, Y., Deniz, V., Bozkurt, V., \& Caglar, M. F. (2016). The evaluation of grinding process using artificial neural network. International Journal of Mineral Processing, 146, 46-53. https://doi.org/10.1016/j.minpro.2015.11.013

[9] Griffin, J. \& Chen, X. (2016). Real-time simulation of neural network classifications from characteristics emitted by acoustic emission during horizontal single grit scratch tests. Journal of Intelligent Manufacturing, 27(3), 507-523. https://doi.org/10.1007/s10845-014-0883-x 
[10] Zafar, T., Kamal, K., Sheikh, Z., Mathavan, S., Ali, U., \& Hashmi, H. (2017). A neural network based approach for background noise reduction in airborne acoustic emission of a machining process. Journal of Mechanical Science and Technology, 31(7), 3171-3182. https://doi.org/10.1007/s12206-017-0606-2

[11] Liu, C. J., Ding, W. F., Li, Z., \& Yang, C. Y. (2017). Prediction of high-speed grinding temperature of titanium matrix composites using BP neural network based on PSO algorithm. International Journal of Advanced Manufacturing Technology, 89(5-8), 2277-2285. https://doi.org/10.1007/s00170-016-9267-z

[12] Simunovic, G., Svalina, I., Simunovic, K., Saric, T., Havrlisan, S., \& Vukelic, D. (2016). Surface roughness assessing based on digital image features. Advances in Production Engineering \& Management, 11(2), 93-104. https://doi.org/10.14743/apem2016.2.212

[13] Tamang, S. K. \& Chandrasekaran, M. (2015). Modeling and optimization of parameters for minimizing surface roughness and tool wear in turning $\mathrm{Al} / \mathrm{SiCp} \mathrm{MMC}$, using conventional and soft computing techniques. Advances in Production Engineering \& Management. 10(2), 59-72. https://doi.org/10.14743/apem2015.2.192.

[14] Šarić, T., Šimunović, G., \& Šimunović, K. (2013). Use of Neural Networks in Prediction and Simulation of Steel Surface Roughness. International journal of simulation modelling, 12(4), 225-236. https://doi.org/10.2507//JSIMM12(4)2.241

[15] Šarić, T., Lujić, R., \& Šimunović, G. (2005). Applying of Artifical Neutral Network in Maintenance Planning of Metallurgical Equipment. Metalurgija, 44(2), 107-112.

\section{Contact information:}

Tomislav ŠARIĆ, PhD, Full Professor

(Corresponding author)

Mechanical Engineering Faculty,

Trg I. B. Mažuranić 2, HR-35000 Slavonski Brod, Croatia

E-mail: tsaric@sfsb.hr

Goran ŠIMUNOVIĆ, PhD, Full Professor

Mechanical Engineering Faculty,

Trg I. B. Mažuranić 2, HR-35000 Slavonski Brod, Croatia

E-mail: gsimun@sfsb.hr

Đorđe VUKELIĆ, PhD, Associate Professor

Faculty of Technical Sciences,

Trg Dositeja Obradovića 6, RS-21000 Novi Sad, Serbia

E-mail: vukelic@uns.ac.rs

Katica ŠıMUNOVIĆ, PhD, Full Professor

Mechanical Engineering Faculty,

$\operatorname{Trg}$ I. B. Mažuranić 2, HR-35000 Slavonski Brod, Croatia

E-mail: ksimun@sfsb.hr

Roberto LUJIĆ, PhD, Full Professor

Mechanical Engineering Faculty,

Trg I. B. Mažuranić 2, HR-35000 Slavonski Brod, Croatia

E-mail: rlujic@sfsb.hr 